\author{
International Journal of Innovative Research in \\ Electrical, Electronics, Instrumentation and Control Engineering
}

Vol. 9, Issue 12, December 2021

DOI: 10.17148/IJIREEICE.2021.91203

\title{
Design and Fabrication of Anti-Drone System with Interdiction Method
}

\author{
Veeramanikandan R ${ }^{1}$, Faisal Hussain Mughloo², Katariya Sagar Bhikubhai ${ }^{3}$, Devika Jayaraj ${ }^{4}$, \\ Nuvvula Yeshawo Narayana ${ }^{5}$
}

Assistant professor, Department of Aeronautical Engineering, Hindusthan College of Engineering and Technology, Coimbatore, India ${ }^{1}$

Student, Department of Aeronautical Engineering, Hindusthan College of Engineering and Technology,

$$
\text { Coimbatore, India }{ }^{2,3,4,5}
$$

\begin{abstract}
In recent years, drones have undergone tremendous development. Due to low price and ease of use, drones have been widely utilized in many application scenarios, which potentially pose great threats to public security and personal privacy. To mitigate these threats, it is necessary to deploy an anti-drone system in the sensitive area to detect, localize and defend against the intruding drones. In this paper, a comprehensive technology utilized for drone surveillance implemented to the existing anti drone systems. A counter drone system includes a cueing sensor to detect the presence of an object wherein the cueing sensor cues the presence of a target drone. A long-range LIDAR system having a sensor pointed in a direction of the target drone to acquire and track at long range the target drone to provide an accurate location of the target drone. The motion of the target drone used to maintain the track of the target drone and a threat detector. The LIDAR data augmented to determine the target drone.
\end{abstract}

Keywords: LIDAR, Cueing Sensors, Anti-Drone, Intruding Drones.

\section{INTRODUCTION}

Unmanned Air Systems (UAS), sometimes known as drones, have been one of the most important technical advancements in recent years, representing a paradigm change in military power. Detecting the presence of a drone, finding it, identifying it and its aim, and then supporting or deploying actions (effectors) to neutralise its effect are all required to reduce or eliminate the danger presented by a hostile UAS. Despite this, the widespread deployment of drones poses significant risks to public safety and personal privacy. An attacker could, for example, strap explosives or other dangerous materials to a drone to carry out an attack; criminals could use drones to smuggle illicit materials across the border; and an operator could command a drone carrying a high-resolution camera to fly over walls and spy on residents' personal information.

Drone surveillance, however, is a difficult task due to the drones' tiny size and poor flying speed at low altitude, and several technologies, such as radar surveillance, audio surveillance, video surveillance, and radio frequency (RF) surveillance, offer promise for drone identification and localization. Each technology has advantages and disadvantages. On the basis of one or more of these technologies, a few anti-drone systems have been created. The performance of these systems is determined by the technologies used, and their application scenarios are likewise determined by the technologies used. Furthermore, not all existing systems can detect, locate, and defend drones at the same time.

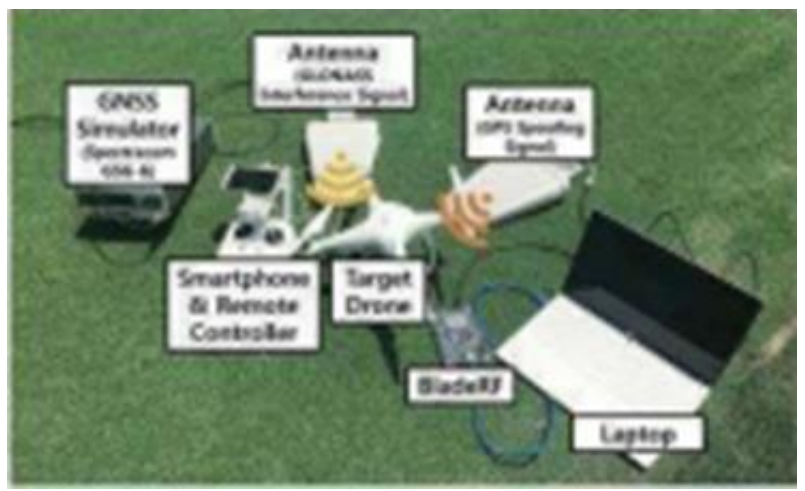

Fig. 1 Tractor beam 
International Journal of Innovative Research in

Electrical, Electronics, Instrumentation and Control Engineering

Vol. 9, Issue 12, December 2021

DOI: $10.17148 /$ IJIREEICE.2021.91203

\section{SURVEILLANCE TECHNOLOGIES AND ANTI-DRONE SYSTEMS}

\section{Surveillance Technologies}

Radar:

Radar is a valuable device for identifying and following of huge airplane, though it faces serious difficulties in distinguishing and following robots, since drones have a low radar cross area and generally fly with low speed at low height. All things considered, radar reconnaissance is promising in distinguishing and following robots. It has been checked that, by investigating the miniature Doppler marks got by multi static radar, the messiness/target separation can be improved, which empowers drone recognition and following high precision.

\section{Audio:}

During the trip of robots, the sounds created by the engines and quick turning propellers can be used in identification, arrangement, and limitation of robots by a framework outfitted with acoustic sensors. The acoustic marks of robots can be acquired by means of investigation in both time and recurrence areas.

Video:

Drone detection based on video images is essentially an object detection problem in the area of computer vision and pattern recognition. An object can be detected based on its appearance features, i.e., colour, contour lines, geometric forms or edges, etc. However, such method might fail when the bird is gliding. For drone detection, it is promising to combine both motion features and appearance features, which would enable the detection with higher accuracy. On the other hand, even though object detection and tracking based on video images have been well investigated, they still suffer from many issues, e.g., occlusions, which also exist in drone detection and tracking.

\section{RF:}

The existing drones in the market or customized drones usually communicate with their controllers at some specific frequency bands, e.g. $2.400-2.483 \mathrm{GHz}$ and $5.725-5.825 \mathrm{GHz}$.

\section{LITERATURE SURVEY}

\section{A. COUNTER-DRONE SYSTEMS}

Recently, commercial drones have become available for the broad public. The prices dropped and the drones became much easier to operate. This lowered the hurdle of entering drone flight, but not only for hobbyists. This creates an enormous potential of hazard. Starting from people trying to spy governmental actions down to kamikaze drones carrying small bombs. To motivate the presented tracking and jamming system, an analysis of the drone control link, the drone behaviour and the already existing systems is done.

\section{Anti-Drone System with Multiple Surveillance Technologies: Architecture, Implementation, and Challenges}

A few anti-drone systems have been developed based on one or several of these technologies. Performances of these systems rely on the utilized technologies, while their application scenarios also vary with the utilized technologies. Moreover, not all the existing systems can simultaneously realize drone detection, localization, and defence.

In this article, we will comprehensively review a few of the most widely used technologies in drone surveillance and some existing anti-drone systems. Then we build up an anti-drone system by fusing multiple surveillance technologies to conduct drone detection, localization and RF jamming. Furthermore, challenges and open research issues are discussed

\section{A Surveillance System for Drone Localization and Tracking Using Acoustic Arrays}

In this paper, we develop a systematic method for drone localization and tracking by using acoustic arrays. Specifically, we develop a time difference of arrival (TDOA) estimation algorithm based on Gauss priori probability density function to overcome the multipath effect and the low signal-to-noise ratio (SNR), and design a localization method by making full use of the TDOA estimation results, followed by tracking the drone by Kalman filter. Finally, field experiments are carried out to verify the effectiveness and performance of the proposed methods. The results show that they can achieve good performance in drone localization and tracking.

\section{Low-Cost Jamming System Against Small Drones Using a 3-D MIMO Radar Based Tracking}

Recently, commercial drones have become available for the broad public. The prices dropped and the drones became much easier to operate. It consists of a 3D Frequency Modulated Continuous Wave (FMCW) Multiple Input Multiple Output (MIMO) radar and a directional jammer working at $2.4 \mathrm{GHz}$. The radar is continuously scanning and waits until a target enters to secure. With the use of directional antenna, the control signal of the drone can be superimposed by a strong jamming signal which makes controlling of the drone impossible. 
International Journal of Innovative Research in Electrical, Electronics, Instrumentation and Control Engineering

Vol. 9, Issue 12, December 2021

DOI: $10.17148 /$ IJIREEICE.2021.91203

\section{III.METHODOLOGY}

\section{A. VARIOUS INTERDICTION METHODS}

RF Jamming: Disrupts the radio frequency link between drone and its operator by generating large volumes of RF output. Once the RF link, which can include can include WIFI links, is served, a served, a drone will either descend to the ground or initiate a "return to home" maneuver.

Nets: Designed to entangle the targeted drone and/or its rotors. Drones are deployed for catching other small drones for the respective action of either defence or counter attack measure.

Crackers: Designed to act like a gun trigger system for counter attack the targeted drone. It is a efficient method for counter attacking enemy drone into our respective surveillance territory.

Combined Interdiction Elements: A number of C-UAS systems also employ a combination of interdiction elementsmost commonly, RF and GNSS jamming systems that work in tandem. In this section we develop an anti- drone system, called ADS-ZJU, which combines three surveillance technologies.

Heterogeneous sensing unit: In the heterogeneous sensing unit, as shown in Fig. 2(a)(b)(c), three kinds of sensors are adopted to capture the information for drone detection and localization:

(a) The utilized acoustic sensor is Type CHZ-213 1/2-inch free-field microphone with Type YG- 201 pre amplifier. We build up L-shape acoustic arrays with four acoustic sensors uniformly installed along each side;

(b) The utilized optical camera is HIKVISION DS-2DF7330IW Network PTZ camera, which supports 360-degree horizontal rotation, -2 90-degree vertical rotation. It can achieve both automatic and manual focusing; (c) The utilized RF sensor is USRP-2955, one kind of software defined radio, which has 4 independently tuneable RX channels and can receive RF signals between $10 \mathrm{MHz}$ and $6 \mathrm{GHz}$. Acoustic signals, video images and RF signals will be collected via these sensors and sent to the central processing unit.

Central processing unit: The central processing unit is the key part of ADS-ZJU, it conducts drone feature extraction, drone detection and drone localization.

Drone feature extraction: Drone feature extraction is essential for drone detection. By taking DJI Phantom 4 as an example, we analyse the drone's features based on the received acoustic signals, video images and RF signals.

Acoustic feature: The spectrum feature of the received acoustic signals is extracted via Short Time Fourier Transform (STFT). Fig. 3(a) shows the STFT result of the acoustic signals generated by a flying DJI Phantom 4. In this figure, different colours represent the strengths of acoustic signals at different frequencies. The unit of the strength values is $\mathrm{dB}$. We can see that the acoustic signals generated by DJI Phantom 4 are harmonic signals with fundamental frequency at about $180 \mathrm{~Hz}$. Strengths of the harmonics are different from each other yet their distribution is quite stable. Therefore, we take the distribution of the harmonics' strengths as the drone's acoustic feature. The feature vector is computed as, the fundamental frequency and harmonics are $180 \mathrm{~Hz}, 360 \mathrm{~Hz}, 540 \mathrm{~Hz}, 720 \mathrm{~Hz}$, etc.

Image feature: We take Histograms of Oriented Gradients (HOG), one of the most effective appearance feature descriptors in object detection, to describe the image feature of drones. Since a drone only occupies a small part of an image, the HOG feature of an image window is utilized in describing the feature of a drone.

Drone detection: The linear support vector machine (SVM) is utilized in drone detection. Before online detection, we will firstly conduct off- line training to obtain drone classifiers, which can separate the drone's feature vectors from other objects' feature vectors. We collect a large amount of acoustic signals, video images and RF signals from both the drone and other objects.

\section{B. DIMENSION OF DRONE}

I. Length of the arm $=450 \mathrm{~mm}$.

II. Length of vertical, horizontal and diagonal sides of base

plate $=43 \mathrm{~mm} \& 35 \mathrm{~mm} .16$

III. Propeller 10 inch

IV. Motor $1000 \mathrm{kv}$ 
International Journal of Innovative Research in Electrical, Electronics, Instrumentation and Control Engineering

Vol. 9, Issue 12, December 2021

DOI: 10.17148/IJIREEICE.2021.91203

V. Receiver 6 channel rf range 2.408-2.475 Ghz, Band 13 , Power 4-6.5v.

VI. Transmitter 6 channel rf range 2.408-2.275 Ghz, Band 135, Power 6v.

\section{COMPONENTS}
a) Frame
b) Control board APM original
c) Standard propellers
d) Electronic Speed Controllers(ESC)
Skywalker-20A
Size- $42 * 2 * 8$
e) Transmitter (6 channel)
FS-i6
f) The Receiver(6 channel)
g) $3200 \mathrm{MaH}$ LiPo Battery
h) NAZA-M Lite
i) Landing gear

Component specifications: NAZA-MLite (All-In-One Design)

For entry-level enthusiasts, DJI now brings out the most cost-effective solution, the NAZA-M Lite flight control system. As the simplified version of NAZA-M, it inherits the high reliability and stability of NAZA-M. The innovative All-inone design simplifies installation and saves space and weight. It contains inner damping, controllers, 3-axis gyroscope, 3-axis accelerometer and barometer in its light and small Main Controller. It can measure flying altitude, attitude and therefore can be used for autopilot/automatic control.

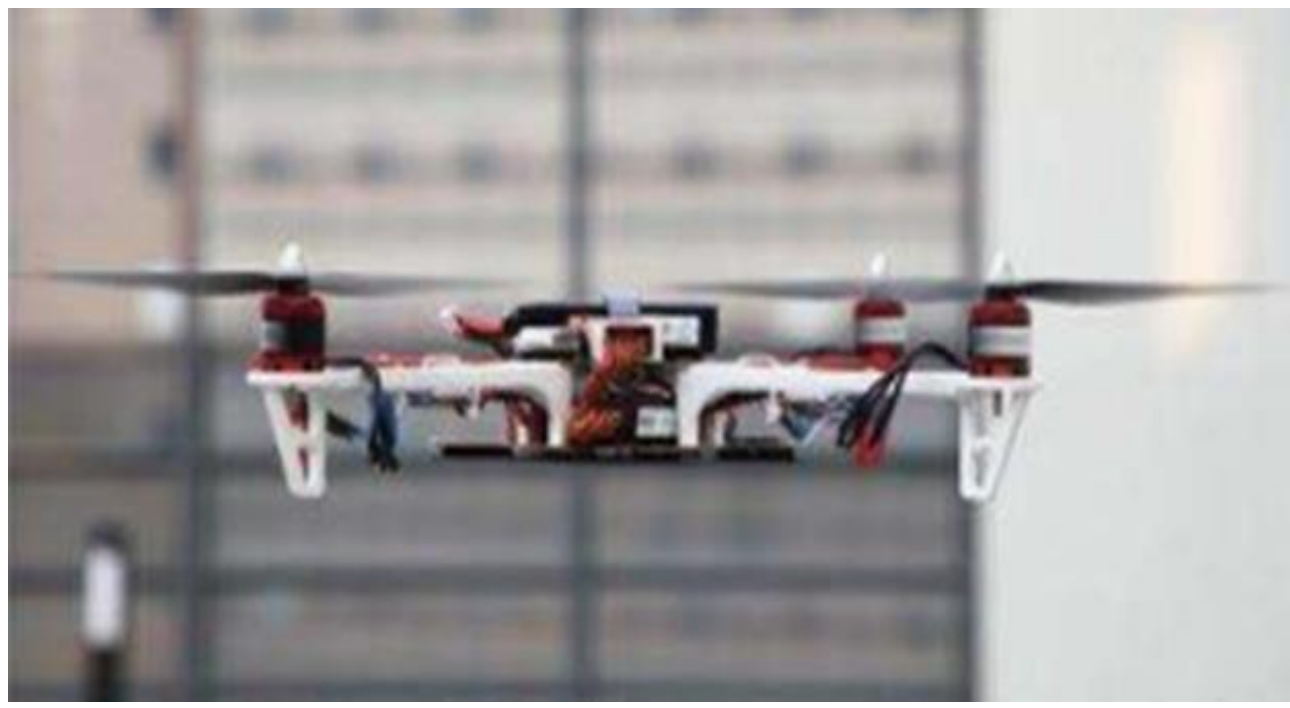

Fig. 2 Drone Controller

\section{Advanced Attitude Stabilize Algorithm}

The advanced attitude stabilization algorithm not only inherits the outstanding flight stability of DJI products, but also provides excellent manoeuvrability. It is more flexible and stable, and gives the hobbyists a wonderful flight experience.

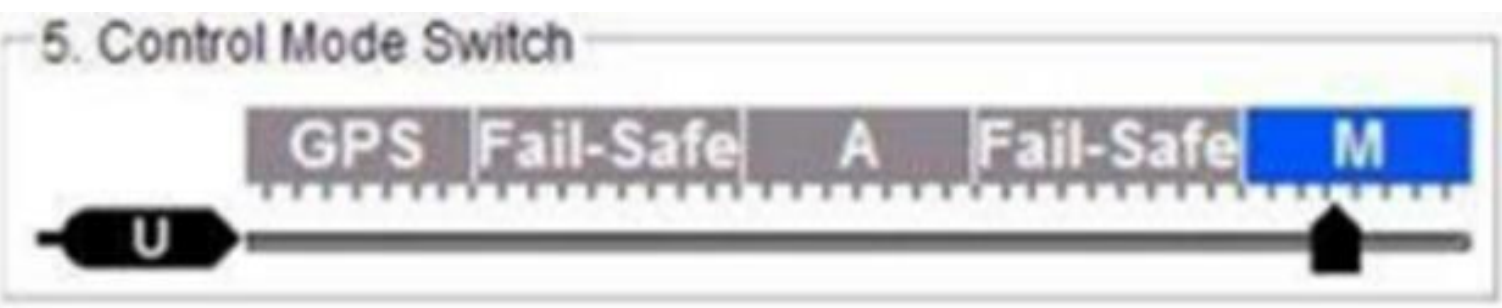

Fig. 3 control mode switch 
International Journal of Innovative Research in Electrical, Electronics, Instrumentation and Control Engineering

Vol. 9, Issue 12, December 2021

DOI: $10.17148 /$ IJIREEICE.2021.91203

Multiple Flight Control Mode/Intelligent Switching:

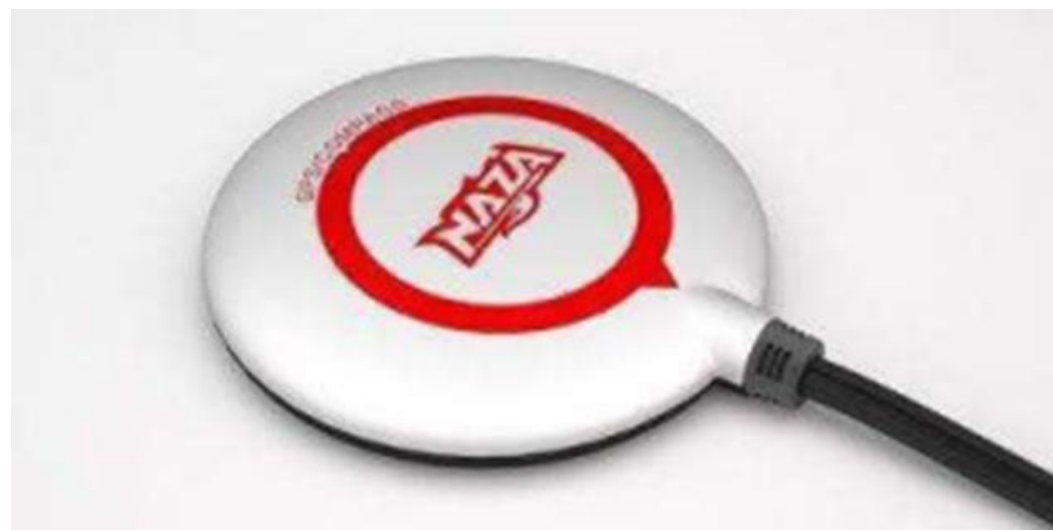

Fig. 4 GPS Compass

GPS Module Available/Accurate Position Hold:

The plug and play GPS module will greatly enhance the performance for Aerial Photography with accurate Position Hold, Return-To-Home and Intelligent Orientation Control functionalities. With the GPS Module, the multi-rotor will have position and altitude locked accurately even in windy conditions. Hovering accuracy is approximately $2.5 \mathrm{~m}$ horizontal and $0.8 \mathrm{~m}$ vertical.
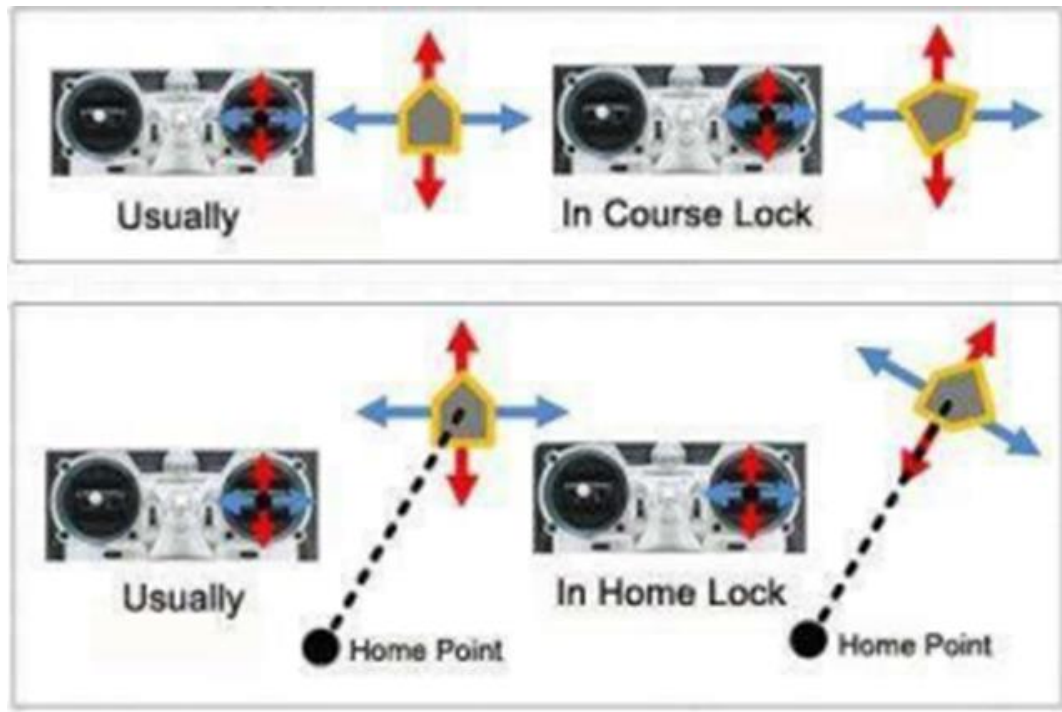

Fig. 5 Controller View

\section{Intelligent Orientation Control (IOC):}

Usually, the forward direction of a flying multi-rotor is the same as the nose direction. By using Intelligent Orientation Control (IOC), wherever the nose points, the forward direction has nothing to do with nose direction:

In course lock flying, the forward direction is the same as a recorded nose direction. See the following figures (Mode 1) : In home lock flying, the forward direction is the same as the direction from home point to multi-rotor. See the following figures (Mode 2) : 
International Journal of Innovative Research in Electrical, Electronics, Instrumentation and Control Engineering

Vol. 9, Issue 12, December 2021

DOI: 10.17148/IJIREEICE.2021.91203

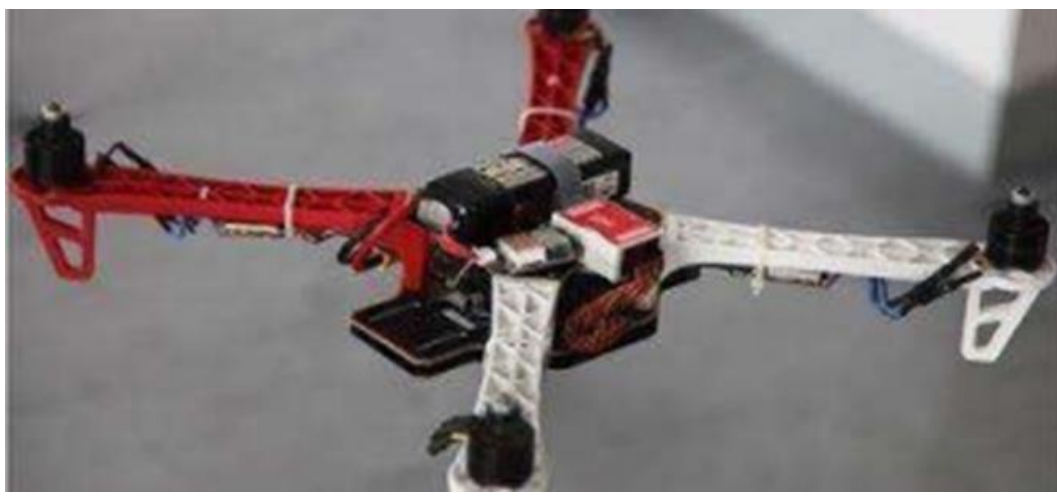

Fig. 6 Drone Assembly

\section{Failsafe Mode}

If your transmitter supports failsafe, then you can set failsafe through port-U. Naza controller has built-in auto level failsafe function, which means when the communication between MC and the transmitter is disconnected, the outputs of all command sticks from controller will go to centre position. If the GPS module is used,

you can also set RTH on failsafe. If your transmitter has only 4 channels, then MC will work in Atti. Mode by default without the failsafe function.
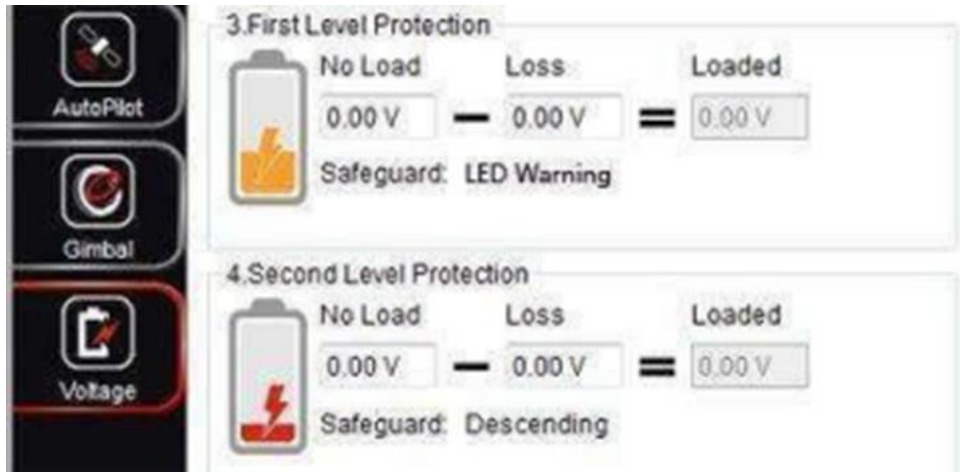

Fig. 7 Drone Battery Protection Level

\section{Low Voltage Protection}

In order to prevent your multi-rotor from crashing or other harmful consequences caused by low battery voltage, we have designed two levels of low voltage protection. You can choose not to use this; however, we strongly recommend you to enable the protection. Both levels of protection have LED warning as default. The first level will blink the red light continuously; the second level will blink red light continuously and the multi-rotor will descend and land. In manual mode when low voltage protection is triggered the LED warning will be active only.

\section{Motor Arm and Motor Dis-Arm}

There are four ways to start the motors, see the following picture:

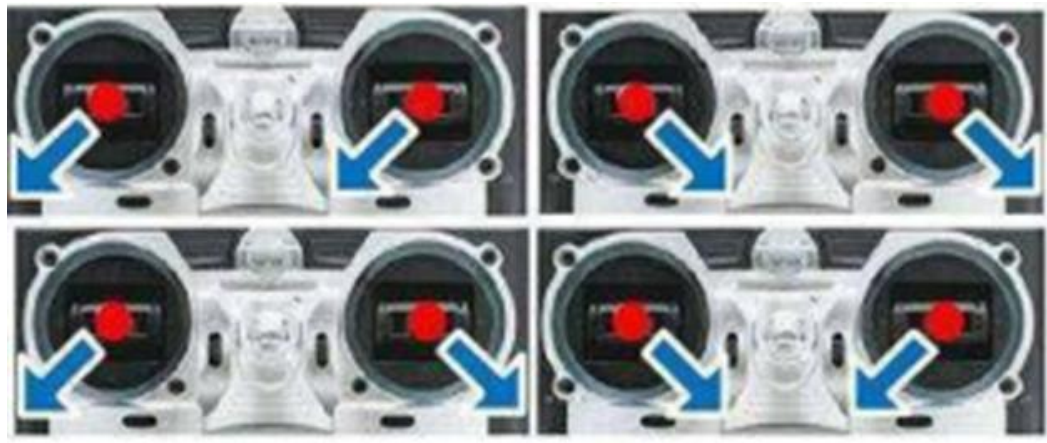

Fig. 8 Drone Controller Moves with Joystick 
International Journal of Innovative Research in Electrical, Electronics, Instrumentation and Control Engineering

Vol. 9, Issue 12, December 2021

DOI: $10.17148 /$ IJIREEICE.2021.91203

When you want to start the multi-rotor, you need to perform any of the ways of CSC. During the flight, if the motors stop, you can immediately execute the CSC and the motors will start. This enhances the safety of the multi-rotor; no uncontrolled starting of the motors.

Motor Disarm: There are two modes of Motor Disarm: namely Intelligent Mode and Immediate Mode. For more information, please refer to the user manual or DJI Wiki.

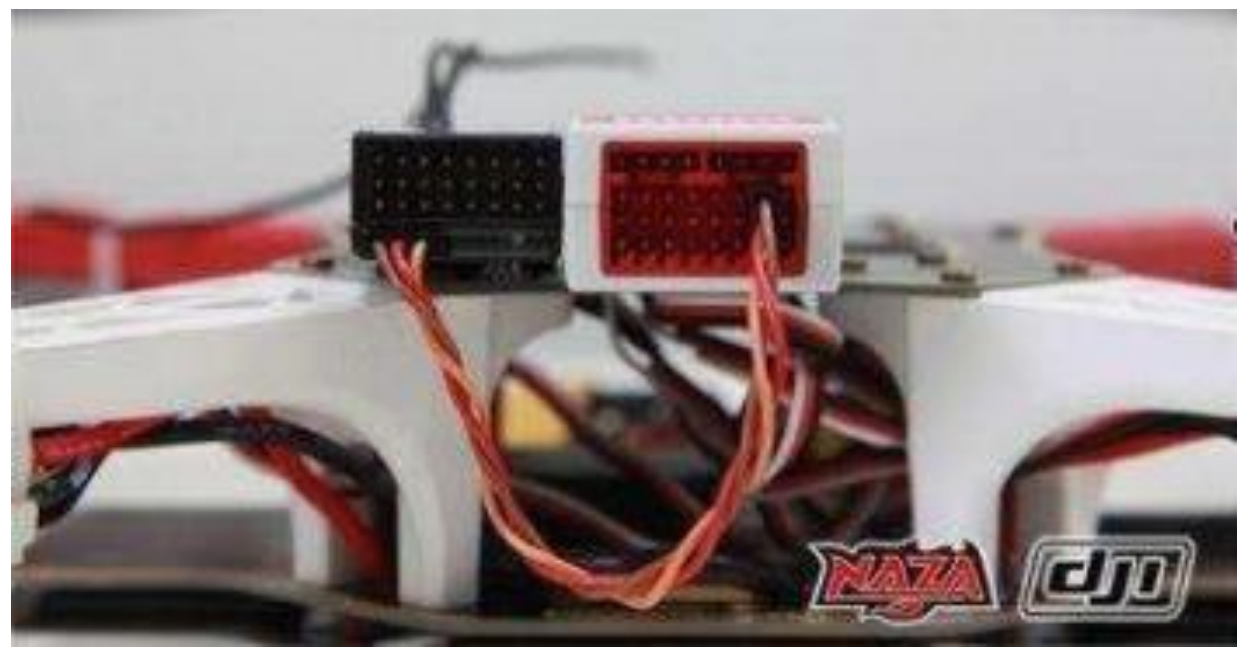

Fig. 9 NAZA Board

\section{Support Futaba S-Bus and PPM Receiver:}

Minimum Four channel receiver supported, also PPM and Futaba S Bus receiver supported.

PPM \& S-Bus receivers (the general use of the first 8 channels of S Bus receiver) to optimize the channel connection, the A, E, T, R, U five-channel functions are set using channel X2, use only one servo cable to connect the PPM or SBus to X2, this makes installation quick and easy, not easy to make mistakes.

\section{Supported Multi-Rotor Types:}

Naza-M Lite now supports six types of multi-rotor, which meets the different needs of the enthusiasts.

Quad I, Quad X;

Hexa I, Hexa V, Hexa Y, Hexa IY;

\section{Built-In Gimbal Stabilization Function:}

The gimbal stabilization module is compatible with almost all 2 -axis gimbal systems. The system will adjust the gimbal and camera according to the attitude of the aircraft after setting the parameters the first time.

\section{Remote Adjustment}

The default parameter settings are OK for you to achieve a normal flight, and it also supports remote parameter adjustment by using a control slider on the TX during flight, in order to obtain better performance.

\section{TABLE I SKYWALKER 20A}

\begin{tabular}{|l|l|l|}
\hline \multicolumn{2}{|l|}{ Specifications of Skywalker 20A } \\
\hline Sl.no. & Class & $\mathbf{2 0 A}$ \\
\hline 1. & Model & Skywalker- 20A \\
\hline 2. & Cont.Current & $20 \mathrm{~A}$ \\
\hline 3. & Burst Current $(>10 \mathrm{~s})$ & $25 \mathrm{~A}$ \\
\hline 4. & BEC.Mode & Linear \\
\hline
\end{tabular}


International Journal of Innovative Research in Electrical, Electronics, Instrumentation and Control Engineering

Vol. 9, Issue 12, December 2021

DOI: 10.17148/IJIREEICE.2021.91203

\begin{tabular}{|l|l|l|l|}
\hline 5. & \multicolumn{2}{|l|}{ BEC Output } & $5 \mathrm{~V} / 2 \mathrm{~A}$ \\
\hline 6. & \multirow{2}{*}{ BEC } & $2 \mathrm{~S}$ & 5 Servos \\
\hline 7. & & $3 \mathrm{~S}$ & 4 Servos \\
\hline 8. & \multirow{2}{*}{ Battery Cell } & Lipo & $2-3 \mathrm{~S}$ \\
\cline { 4 - 5 } 9. & & NiM H & $5-9$ Cells \\
\hline 10. & Weight & $\mathrm{L} * \mathrm{~W}$ & $42 * 25 * 8$ \\
\hline 11. & Size & & $19 \mathrm{~g}$ \\
\hline
\end{tabular}

\section{Specification}

Output: Continuous 20A, Burst 25A up to 10 seconds.

Input Voltage: 2-3S Lipo, 5-9 cells NiMH.

BEC: 2A / 5V Linear mode BEC.

Refresh rate of the throttle signal: $50 \mathrm{~Hz}$ to $432 \mathrm{~Hz}$.

Max Speed: 210000rpm for 2 Poles BLM, 70000rpm for 6 poles BLM, 35000rpm for 12 poles BLM.

(BLM = Brushless Motor)

Size: $42 \mathrm{~mm} * 25 \mathrm{~mm} * 8 \mathrm{~mm}$.

Weight: $19 \mathrm{~g}$.

\section{Features}

Safety arming feature: Regardless the throttle stick position, the motor will not spin after battery connected.

Throttle calibration function: Throttle range can be calibrated to be compatible with different transmitters.

User programmable.

Multiple program methods supported: transmitter stick, LED Program Card. Very easy to program the ESC at home or at the flying field.

Compatible with Lipo and NiMH battery.

Timing can be changed to be suitable for different brushless motors.

Full protection: Low voltage cut-off protection / Over-heat protection / Throttle signal loss protection.

\section{Numerical Analysis}

wGL- Ground lift weight

$w G L-1301 g+300 g$

WGL $-1601 \mathrm{~g}$

For a quad copter the required thrust $1601 \mathrm{~g}$ which is the ground lift weight of the drone.

For an ideal quad copter to fly the TWR must be greater than 2 TWR > 2

Each quad copter motor should produce thrust that lift half the weight of the drone.

Since WGL = $1601 \mathrm{~g}$

The total required thrust is $1601 \times 2=3202 \mathrm{~g}$.

\section{Weight Estimation:}

Weight of the components:

Q450-Frame weight $=330 \mathrm{~g}$

Q450-Landing gear weight $=300 \mathrm{~g}$

Flight controller $=50 \mathrm{~g}$

ESC weight $=19 \times 4=76 \mathrm{~g}$

Motor weight $=80 \mathrm{x} 4=320 \mathrm{~g}$

Battery $=215 \mathrm{~g}$ Receiver $=10 \mathrm{~g}$

General Weight Equation:

$\mathrm{WGL}=\mathrm{WE}+\mathrm{WF}+\mathrm{WPL}+\mathrm{WCREW}$ 
International Journal of Innovative Research in Electrical, Electronics, Instrumentation and Control Engineering

Vol. 9, Issue 12, December 2021

DOI: 10.17148/IJIREEICE.2021.91203

WE -Empty weight

$\mathrm{WE}=$ Total weight of the components

$\mathrm{WE}=1301 \mathrm{~g}$

For quad copter WF \& WCREW is zero

WPL $=$ Setup weight + Cracker weight

$\mathrm{WPL}=300 \mathrm{~g}$

Therefore, the thrust required for each motor

$=$ Total thrust $/$ No. of motors

$=3202 / 4$

$=800 \mathrm{~g}$ (approx.)

Since we have used $1000 \mathrm{kv}$ brush-less DC motor with a 9x4.5 APC propeller each motor is efficient to lift 916g of weight (The $1000 \mathrm{kv}$ refers to the $1000 \mathrm{rpm}$ per 1 volt of current) Our battery is efficient to deliver $11.1 \mathrm{v}$ of current Therefore maximum rpm without any load on the motor is $11100 \mathrm{rpm}$.

But efficiently the lithium polymer battery can supply $80 \%$ of current which is $9.7 \mathrm{v}$.

Therefore, the rpm of each motor $=9700 \mathrm{rpm}$.

\section{Battery life estimation:}

For lipo battery the best equation to find the life of battery is $t=\eta \times 100060 \times \mathrm{c} / 4 \times \mathrm{v} / \mathrm{p}$

28

Where $\eta=$ efficiency factor for energy loss for all

quad-copter is $0.8 \mathrm{c}=$ battery capacity in mah. $\mathrm{v}=$ voltage for battery. $\mathrm{p}=$ power required for

each battery.

$\mathrm{p}=1(2 \mathrm{mg}) 3 / 2$

$4 \sqrt{ } \rho \mathrm{A}$

Where $p=$ air density $=1.2 \mathrm{~kg} / \mathrm{m}^{2}$.

$\mathrm{A}=$ rotor area.

$\mathrm{m}=$ mass of drone $\&$ payload.

$\mathrm{g}=$ gravity $=9.81$

$21.6 \mathrm{z} 9.813 / 2$

Time period:

$\mathrm{p}=1$

$41.201270127 \mathrm{p}=178.32$ watts

$\mathrm{t}=0.8 \quad 1000 \mathrm{t}=2.4$

\section{CONCLUSION}

A comprehensive review of four of the most widely used surveillance technologies in drone detection are briefly discussed also summarized existing anti-drone systems. There are several advantages and drawbacks for each method with has taken from reference article and the suitable method for specific surrounding are taken to consideration, an anti-drone is assembled and tested.

A drone with cracker as a payload which can be used either as defending purpose for civilization purpose and for counter attacking enemy drones in attacking purpose. It can localize the intruding drone in a campus environment by the modern ADS system and also for continuous monitoring, a counter drone system includes a semi-autonomous response planner system with inputs from the threat detector and an operator to determine countermeasures appropriate for the cued target drone.

Furthermore, the challenges and open research on countermeasures can be determined and implemented to counter any threats by the target drone. In accordance with the disclosure, a method includes: detecting a presence of a target drone using a cuing sensor; acquiring, in response to initial data from the cuing sensor, a target drone and then tracking the target drone using a long

range LIDAR system to provide an accurate location of the target drone and to maintain the track of the target drone. It has been tested that an anti-drone with suitable interdiction method is used as efficient way in military as well as economical purpose. 
International Journal of Innovative Research in Electrical, Electronics, Instrumentation and Control Engineering

Vol. 9, Issue 12, December 2021

DOI: 10.17148/IJIREEICE.2021.91203

\section{REFERENCES}

[1]. 1. Mellinger, D., Michael, N., Kumar, V.: Trajectory generation and control for precise aggressive maneuvers with quadrotors. In: International Symposium on Experimental Robotics, Delhi, India (2010).

[2]. 2. Achtelik, M., Bachrach, A., He, R., Prentice, S., Roy, N.: Stereo vision and laser odometry for autonomous helicopters in GPS-denied indoor environments. In:SPIE Unmanned Systems Technology XI. Volume 7332., Orlando, F (2009).

[3]. 3. Bl"oandsch, M., Weiss, S., Scaramuzza, D., Siegwart, R.: Vision based MAV navigation in unknown and unstructured environments. In: IEEE Int. Conf. on Robotics and Automation. (2010) $21-28$

[4]. 4. Michael, N., Fink, J., Kumar, V.: Cooperative manipulation and transportation with aerial robots. Autonomous Robots 30 (2011) 73-86.

[5]. 5. Multicopter: List of helicopter projects (2011) http://multicopter.org/wiki/.

[6]. 6. Bills, C., Chen, J., Saxena, A.: Autonomous MAV flight in indoor environments using single image perspective cues. In: IEEE Int. Conf. on Robotics and Automation. (2011)

[7]. 7. Bills, C., Yosinski, J.: MAV stabilization using machine learning and onboard sensors. Technical Report CS6780, Cornell University (2010)

[8]. 8. Faigl, J., Krajn'ik, T., Von'asek, V., P`reu cil, L.: Surveillance planning with localization uncertainty for mobile robots. In: 3rd Israeli Conference on Robotics.(2010)

[9]. 9. Ng, W.S., Sharlin, E.: Collocated interaction with flying robots. Technical Report 2011-998-10, Dept. of Computer Science, University of Calgary, Canada (2011)

[10]. 10. Higuchi, K., Shimada, T., Rekimoto, J.: Flying sports assistant: external visual imagery representation for sports training. In: 2 nd Augmented Human International Conference, New York, NY, USA, ACM (2011) 7:1-7:4

[11]. 11. Krajn'ık, T.: Simple 'getting started' applications for AR-

[12]. 29

[13]. drone (2011) http:// labe.felk.cvut.cz/ tkrajnik/ardrone. 12. Solc, F.: Modelling and control of a quadrocopter. 13. Kailath, T.: Linear Systems. Prentice Hall (1980) 14. Krajn'1k, T., Faigl, J., Von'asek, V., Ko`snar, K., Kulich, M.

[14]. P`reu`cil, L.: Simple yet stable bearing-only navigation. Journal of Field Robotics 27 (2010) 511-533

[15]. 15. Bay, H., Ess, A., Tuytelaars, T., Van Gool, L.: Speeded-Up Robust Features (SURF). Computer Vision and Image Understanding 110 (2008) 346-359

[16]. 16. Saska, M., Von'asek, V., P`reu čcil, L.: Roads sweeping by unmanned multi-vehicle formations. In: IEEE Int. Conf. on Robotics and Automation. (2011)

[17]. 17. Consolini, L., Morbidi, F., Prattichizzo, D., Tosques, M.: Leader-follower formation control of nonholonomic mobile robots with input constraints. Automatica 44 (2008) 1343-1349

18. Spitz, S.N., Requicha, A.A.G.: Multiple-Goals Path Planning for Coordinate Measuring Machines. In: IEEE Int. Conf. on Robotics and Automation. (2000) 